AIAA-2001-3781

Grid Erosion Analysis of

The T5 Ion Thruster

I. D. Boyd

University of Michigan

Ann Arbor, MI 48109.

M. W. Crofton

The Aerospace Corporation

Los Angeles, CA 90009.

For permission to copy or to republish, contact the copyright owner named on the first page. For AIAA-held copyright, write to AIAA Permissions Department, 1801 Alexander Bell Drive, Suite 500, Reston, VA, 20191-4344. 
AIAA Paper 2001-3781

AIAA 37th Joint Propulsion Conference, Salt Lake City, Utah, July 2001

\title{
GRID EROSION ANALYSIS OF THE T5 ION THRUSTER
}

\author{
Iain D. Boyd* \\ University of Michigan, Ann Arbor, MI 48109 \\ Mark W. Crofton $\dagger$ \\ The Aerospace Corporation, Los Angeles, CA 90009
}

\begin{abstract}
The optics of the three-grid T5 electrostatic ion thruster are analyzed computationally using a combination of a hybrid fluid-Particle In Cell method for the plasma dynamics, and the direct simulation Monte Carlo method for collision dynamics. This is performed by considering the plasma flow through a single, representative aperture. The accuracy of the model is assessed through direct comparison with experimental measurements of currents collected on both the accel and decel grids over a range of thruster operating conditions. Agreement between the predicted and measured data is very good for all conditions studied. The sensitivity of the simulated results to a number of basic assumptions in the model is considered. Detailed data on the properties of the ions impacting on the accel and decel grids are extracted from the simulations and used to explain the macroscopic phenomena.
\end{abstract}

\section{Introduction}

For ion engines, erosion of the accelerator and decelerator grids is a principal life-limiting process and a primary source of thruster-induced spacecraft contamination. ${ }^{1,2}$ The usual grid material molybdenum is sputtered into the ion engine plume. It deposits on neighboring surfaces upon contact, leaving a metallic coating that alters surface properties and may affect the performance of solar arrays, sensors, and thermal control materials.

The limitation of lifetime due to erosion of the grids (primarily the accel grid but also the decel grid) exerts considerable influence on the choice of thruster type and rated thrust for the spacecraft. The end of life is usually taken at the point of grid mechanical failure due to extensive loss of the webbing. This results in loss of beam current capability and the onset of electron backstreaming into the discharge chamber. The erosion of grid material may also produce flakes which electrically short adjacent grids as may have temporarily occurred at the beginning of the Deep Space- 1 mission. ${ }^{3}$ For the more than 60 ion engine endurance tests that have been reported, the majority of failure modes involved the accel grid. ${ }^{1}$ To partially

* Associate Professor. Department of Aerospace Engineering.

$\dagger$ Research scientist. Space Materials Laboratory. 
mitigate grid erosion effects, significant effort has been expended in the development of grids using erosionresistant materials such as carbon-carbon composites ${ }^{4}$ and titanium. ${ }^{5}$

The purpose of the present study is to report on minor modifications to an erosion model presented by Boyd and Crofton. ${ }^{6}$ These modifications concern the simulation of the neutral plasma downstream of the decel grid, and will be described later. In addition to presenting updated predictions of grid currents and grid erosion, the present study will provide detailed information on the characteristics of the ions impacting on the grids. The model employs a combination of a fluid-Particle-In-Cell (PIC) approach ${ }^{7}$ for electrostatics, and the direct simulation Monte Carlo (DSMC) method $^{8}$ for collisions. Collision mechanisms included in the code involve momentum transfer, charge exchange, and Coulomb interactions. The code solves the Poisson equation to determine the potential field.

In the following, the experimental investigation is described briefly. More detailed information is provided in Ref. 9. Then, the computational model is discussed in detail. A variety of results is presented for a range of operating conditions of the thruster. These include general flow field properties, performance (thrust), accel and decel grid currents, ion energy and angle distributions, and grid mass erosion.

\section{Summary of Experimental Results}

Details of the facility at the time of the test were described previously. ${ }^{2}$ The test chamber was $5.5 \mathrm{~m}$ in length and $2.4 \mathrm{~m}$ in diameter. Two custom reentrant cryopumps of combined 70,000 l/s pumping speed on xenon were mounted inside the chamber, suspended from flanges. During thruster operation at a nominal thrust level of $18 \mathrm{mN}$, the chamber pressure was determined to be $1.5 \times 10^{-6}$ Torr, after applying a standard xenon-sensitivity correction to the indicated ion gauge reading. The thruster was mounted on a fixed stand in a side chamber with the ion beam directed across the main chamber. The beam dump consisted of aluminum plates covered with carbon-carbon composite panels, without water cooling. In the experimental study, two extraction grid sets of flight-quality (designated GS003 and GS004) were installed on the T5 ion thruster already characterized by past studies, ${ }^{2}$ to perform design validation. In the present study, we will consider only the data obtained with the GS003 grid set. The current collected during the experiments on GS004 showed a decrease with time. While it is important for modeling efforts to be able to predict such behavior, this lies beyond the scope of the present investigation. The thruster is shown in Fig. 1. The anode was biased at the usual $1100 \mathrm{~V}$ operating point. ${ }^{2}$ The screen grid and discharge chamber wall were about $40 \mathrm{~V}$ lower according to the value of the discharge voltage.

The screen and decel apertures were $2.15-2.18 \mathrm{~mm}$ in diameter, with a hole pitch of about $2.44 \mathrm{~mm}$. The accel aperture diameter was $1.75-1.78 \mathrm{~mm}$ with similar pitch. Grids were $0.25 \mathrm{~mm}$ thick for screen and decel grids, and $1.00 \mathrm{~mm}$ for the accel grid which was spaced $0.75 \mathrm{~mm}$ from the screen grid and $0.50 \mathrm{~mm}$ from the decel grid. Grid set GS003 had been previously operated for a period of roughly 50 hours at thrust levels around $18 \mathrm{mN}$. The mass flow rate was determined from calibrations of shunted flow rates with the engine off, monitoring the rate of pressure rise in a known volume. The absolute accuracy is better than $2 \%$ in each case. It was assumed that the flow rates measured with the engine off were identical to those 
obtained with the engine on.

The thruster characteristics at several operating points are listed in Table 1. The overall propellant mass utilization efficiency, $\eta$, includes both $\mathrm{Xe}^{+}$and $\mathrm{Xe}^{2+}$. The fractional $\mathrm{Xe}^{2+}$ current, $\zeta$, was estimated from measurements by Pollard. ${ }^{10}$

\section{Modeling}

Computer models of flow through ion grid apertures have been developed previously in several investigations. Among the first of these studies, Arakawa and Ishihara ${ }^{11}$ employed a particle description of the ions. Neutral flow was neglected as was charge exchange. In the approach of Peng et al. ${ }^{12}$, the ions were again treated using particles but in this case charge exchange was included by assuming a constant neutral flow field. Bond and Latham ${ }^{13}$ employed a particle approach for the ions, and used curve fits based on a few simulations to describe the spatial distribution of the neutral atom density. In Ref. 13, the computer simulations were employed to optimize the extraction grids for the UK-10 ion thruster that is similar to the T5 thruster studied here. More recent computational studies continue to use a similar approach, e.g. Ref. 14. In Refs. 11-14, only singly charged ions were considered, and the treatment of the neutrals was simplified. In all cases, including the present study, the plasma flow through a single, axially symmetric aperture is considered. Overall effects for the entire grid sets that usually contain thousands of apertures, are simply obtained by scaling up the single aperture results. Note that the $\mathrm{T} 5$ thruster grid sets considered here contain 1,551 apertures.

In the present work, the ions and neutrals are treated in detail using a combination of two particle methods. A hybrid fluid-Particle-In-Cell approach (PIC) ${ }^{6}$ is employed to model the plasma dynamics. In the present study, ions (both single and double charge states) are treated as particles. Following the work of Peng et al. ${ }^{12}$ electrons are only assumed to exist in the regions upstream of the screen grid and downstream of the decel grid. This is a reasonable assumption based on the voltages applied to the grids. A fluid approach is employed for the electrons in which their temperature, $T_{e}$, is assumed to be constant, and their number density, $n_{e}$, is given by the Boltzmann relation:

$$
n_{e}=n_{r e f} \exp \left(\frac{\phi-\phi_{0}}{T_{e}}\right)
$$

where $\phi$ is the local plasma potential and the electron temperature is in units of electron volts. In the region upstream of the screen grid, the reference potential, $\phi_{0}(1)$, is the anode potential $(1100 \mathrm{~V})$, and the reference density, $n_{r e f}(1)$, is the plasma density of the discharge chamber. In the region downstream of the decel grid, the reference potential $\phi_{0}(2)$ is assumed to be $0 \mathrm{~V}$. The reference density, $n_{\text {ref }}(2)$, is the plasma density where the potential in the downstream region becomes equal to $0 \mathrm{~V}$. This is assumed to occur outside the main beam in the back ground plasma of the test facility and is of the order of $10^{14} \mathrm{~m}^{-3}$. Potential probe measurements taken by de Boer ${ }^{15}$ in the plume of the UK-10 thruster indicate that this is a reasonable assumption. 
In the plasma downstream of the computational domain, charge exchange processes create low energy ions that can flow back across the downstream boundary onto the grids. In the present work, the density of these charge exchange ions is assumed to scale with the product of the neutral and plasma densities and can be written:

$$
n_{C E X} \propto \frac{(1-\eta)}{\eta} I_{b e a m}^{2}
$$

These ions flow across the boundary at the most probable thermal velocity given by the assumed background temperature of $300 \mathrm{~K}$. The constant of proportionality in Eq. (2) is obtained for the $399 \mathrm{~mA}$ beam current case by matching the computed to the measured decel grid currents. This same constant is then used to obtain the reference downstream plasma densities for all other flow conditions. The values of $n_{C E X}$ for all operating points investigated are plotted in Fig. 2. It will be found that the variation of these values correlates strongly with the measured grid currents. These downstream boundary conditions differ from those employed in Ref. 6 in two ways. First, in Ref. 6, the density of the back-flowing ions was held constant for all operating conditions. Second, in Ref. 6, the plasma potential was imposed as $0 \mathrm{~V}$ all along the downstream boundary. In the present work, however, the potential there is calculated by assuming charge neutrality, and then Eq. (1) is inverted to evaluate the potential, $\phi$.

The Poisson equation is solved on a rectangular grid where the ion number density is given by the spatial distribution of the particles and the electron number density is given by Eq. (1). Following the approach of Roy et al., ${ }^{16}$ an Alternating Direction Implicit (ADI) method is employed to solve the Poisson equation which is non-linear due to the presence of $\phi$ in Eq. (1) for the electron number density.

The second particle technique employed is the direct simulation Monte Carlo method (DSMC) ${ }^{8}$ which is used to model collision phenomena. Thus, the neutral atoms are also treated as particles, and three different types of collisions are modeled: (1) charge exchange between $\mathrm{Xe}$ and $\mathrm{Xe}^{+}$and between $\mathrm{Xe}$ and $\mathrm{Xe}^{2+}$ using the cross sections measured recently by Pullins et al. ${ }^{17}$; (2) momentum transfer in which the cross sections are given by the model of Dalgarno et al. ${ }^{18}$ and (3) Coulomb collisions. In charge transfer there is no momentum exchanged. In momentum transfer collisions, isotropic scattering in the center of mass frame is assumed. For Coulomb collisions, the equations from classical plasma physics for cross section and scattering angle are employed, e.g., see Ref. 19. However, the scattering angle for a particular collision is sampled statistically from the distribution suggested by Nanbu. ${ }^{19}$ This procedure is necessary because DSMC-PIC is a statistical approach and the method does not compute intersecting particle trajectories deterministically.

The upstream boundary conditions for the DSMC-PIC approach involve specification of velocity distribution functions for each species. Under the assumption of thermal equilibrium in the discharge plasma, this is most conveniently achieved using Maxwell-Boltzmann distributions which require specification of species number densities, velocities, and temperatures. The electron temperature is a very important parameter as it strongly influences electrostatic effects through the Boltzmann relation. It also determines the ion drift 
velocity upstream of the screen grid through the Bohm relation:

$$
V_{i}=\sqrt{\frac{k T_{e}}{m_{i}}}
$$

where $k$ is Boltzmann's constant and $m_{i}$ is the ion mass. A value of $5 \mathrm{eV}$ is assumed for the electron temperature for most of the results presented in this study in the the regions upstream of the screen grid and downstream of the decel grid.

The flux of neutral atoms across a radial surface upstream of the screen grid is given by the standard result from kinetic theory:

$$
\Gamma_{a}=\frac{1}{4} n_{a} V_{a}
$$

where the mean molecular speed is given by:

$$
V_{a}=\sqrt{\frac{8 k T_{a}}{\pi m_{a}}}
$$

In Eqs. (4)-(5), subscript $a$ indicates properties of the atoms. The temperature of the neutral atoms is assumed to be $500 \mathrm{~K}$. The atom and ion fluxes are computed so as to be consistent with the experimentally measured values of total beam current and mass flow rate. Thus, using Eqs. (3)-(5), the atom and ion number densities are determined. One further parameter that must be assigned is $\zeta$, the fraction of ion current represented by $\mathrm{Xe}^{2+}$. In Table 1, estimates are given for $\zeta$ based on the measurements of Pollard ${ }^{10}$.

Finally, the ion temperature must be assigned to determine the distribution of ion velocities flowing into the computational domain. Most of the results presented in this study employ an ion temperature of $3.0 \mathrm{eV}$. This value is consistent with experimental measurements made in an ECR argon plasma ${ }^{20}$ using a plasma density and magnetic field that are similar to those employed in the plasma source of the UK-T5.

The computational grid employed in most of the simulations is shown in Fig. 3. The cell sizes are 20 to $40 \mu \mathrm{m}$, which are of the order of the Debye length for the conditions investigated. These sizes are orders of magnitude smaller than the mean free paths for all flow conditions. The time-step employed in all the simulations is $0.4 \mathrm{~ns}$, and is a fraction of the reciprocal of the plasma frequency. The surfaces of the three grids are at fixed potentials and are assumed to have a constant temperature of $500 \mathrm{~K}$. Ions striking any of the grids are assumed to recombine to neutral atoms. The facility back pressure is sufficiently low $(0.2 \mathrm{mPa})$ such that it should have no effect on the flow through the grids. For completeness, it is included, and is assumed to consist of neutral xenon at a temperature of $300 \mathrm{~K}$.

One of the main results from the computations is the ion current collected by the accel and decel grids. This is computed by directly counting the number of ion particles impacting on any surface of the particular grid over a finite period of physical time.

\section{Results}

\section{General Flow Field Results}

We first consider some general flow field properties for the case with a beam current of $399 \mathrm{~mA}$. In Fig. 4, contours of plasma potential (in Volts) are shown. These show a sheath structure formed in front of 
the screen grid. The structure of the potential field downstream of the decel grid leads to a focusing of ions onto the grid at large radial coordinates, giving rise to the pits and grooves erosion patterns observed on many systems. It is expected that charge exchange ions will play a significant role in determining the grid currents. The volumetric production rate of charge exchange ions is given by:

$$
\nu_{C E X}=n_{i} n_{a}<\sigma_{c e x} g>
$$

where $n_{i}$ is the ion number density, $n_{a}$ is the atom number density, $\sigma_{C E X}$ is the $\mathrm{Xe}^{-} \mathrm{Xe}^{+}$charge exchange cross section, and $g$ is the relative collision velocity. Contours of $\nu_{C E X}$ are shown in Fig. 5 and indicate that the primary region of charge exchange production occurs on the centerline of the beamlet immediately below the accel grid. These contours approximately follow those of the ion density. The charge exchange ions can be attracted to the accel and decel grids due to their relatively low energies.

To illustrate the macroscopic behavior of the computational model, Fig. 6 shows the variation of computed thrust as a function of beam current. The thrust levels shown in Table 1 are nominal values which assume that $\zeta$ and the beam divergence are both zero. They were obtained from the expression

$$
F=I_{\text {beam }} \frac{m}{e} V_{e}
$$

where $V_{e}$ is the exhaust velocity and $m / e$ is the mass to charge ratio. Using $\zeta$ from Table 1 , individual $\mathrm{Xe}^{+}$ and $\mathrm{Xe}^{2+}$ contributions to the thrust are determined. The sum is plotted in Fig. 6 . In both these data and the simulations, the thrust has a nearly linear dependence on beam current. While there is some uncertainty in the specification of the discharge plasma properties, in particular the ion and electron temperatures and the fraction of $\mathrm{Xe}^{2+}$ current, it is encouraging that the thrust predictions are in excellent agreement with the estimated data over the entire range of operating conditions.

\section{Grid Results}

First, we consider the predictions of the total ion current collected on each of the grids. The results for the accel grid are shown in Figs. $7 \mathrm{a}$ and $7 \mathrm{~b}$ as a function of the beam current. As shown in Fig. 7a, the new results obtained with the modified boundary conditions for the downstream plasma are almost identical to the old results reported in Ref. 6 which gave very good agreement with the measured data. The measurements exhibit variation with changes in both beam current and total mass flow rate. It is significant that the model is able to successfully capture these variations. Also included in Fig. 7a, are a few data points for simulations in which there was no backflow of ions across the downstream boundary. These give an indication of the relative contributions to the total accel grid currents from ions originating in the discharge and downstream plasmas. In Fig. $7 \mathrm{~b}$, the sensitivity of the accel grid predictions to the values of the electron temperature assumed in the discharge and downstream plasmas is investigated for several operating conditions. It is found that decreasing either of the electron temperatures leads to decreases in the accel grid current. 
The corresponding results for the decel grid currents are shown in Figs. $7 \mathrm{c}$ and $7 \mathrm{~d}$. In Fig. 7c, it is shown that the new computations with the modified downstream plasma boundary conditions provide significant increases in decel grid current in comparison with the old results, and now offer reasonable agreement with the experimental data. The results obtained with no backflow of ions essentially reproduce the old results. The data shown in Fig. 7d indicate that increases in electron temperature in either the discharge or downstream plasma lead to increases in the decel grid current.

The successful prediction of the grid currents measured on both the accel and decel grids for a range of operating conditions is a significant achievement, but these are macroscopic results. Detailed information about the nature of the ions impacting on the grids is of interest to understand the sputtering processes that accompany this phenomenon. In Figs. $8 \mathrm{a}$ and $8 \mathrm{~b}$, the ion impact energy distribution functions on the barrels of the accel and decel grids are shown at beam currents of 399 and $218 \mathrm{~mA}$, respectively. The general shapes of the energy distributions for all beam currents are found to be similar. For the accel grid, there is a main peak over the range of 100 to $200 \mathrm{eV}$ followed by a wide, decaying structure extending to about $800 \mathrm{eV}$. All of these impacts involve charge exchange ions created at different locations in the plasma flow through the aperture. In both cases shown, there is also a local peak at about 1,300 eV corresponding to direct impacts of primary beam ions. The direct impact peak for the $218 \mathrm{~mA}$ beam current is more than an order of magnitude larger than that for the $399 \mathrm{~mA}$ case. For the decel grid, there is a very low energy peak centered around $50 \mathrm{eV}$. These are assumed to be backflow ions that have been accelerated from zero to the $-50 \mathrm{~V}$ potential of the decel grid. A less populated structure also extends to about $500 \mathrm{eV}$, representing charge exchange ions generated in the intergrid region that missed the accel grid but are collected on the decel grid. For both beam currents shown, there is a significant population of ions centered at about 1,100 eV representing direct impingement of primary beam ions. These decel grid direct impingement peaks are larger than those on the accel grid. Overall, the data shown in Figs. 8a and $8 \mathrm{~b}$ indicate that the populations of ions impacting on the accel and decel grids are different in several ways. The accel grid ions are mainly charge exchange ions with a broad spectrum of energies from 0 to $800 \mathrm{eV}$, and relatively few direct impacts of primary ions. The decel grid ions are mainly backflowing ions from the downstream plasma although there is also a significant population of direct impact ions.

The distributions of the incident angle of ions impacting on the horizontal barrels of the accel and decel grids are shown in Figs. 9a and 9b for beam currents of 399 and $218 \mathrm{~mA}$, respectively. The incident angle is defined such that $0^{\circ}$ corresponds to impact normal (perpendicular) to the surface, positive angles are generated by ions moving from upstream, and negative angles are generated by ions moving from downstream. Once again, the data for all operating conditions are found to have the same general trends. For the accel grid, there are two peaks. One is centered at about $+60^{\circ}$ corresponding to charge exchange ions created in the inter-grid region that are pulled onto the accel grid. The second peak has a smaller population centered around $-50^{\circ}$ representing backflowing ions that missed the decel grid but are subsequently attracted onto the accel grid. For the decel grid, there is a main peak centered around $-50^{\circ}$ corresponding to backflowing ions 
pulled directly onto the decel grid. There is a very small population of ions impacting the decel grid from the upstream plasma. It is well known that the sputter yield of ions on simple materials has a strong angular dependence. ${ }^{21}$ The ability to simulate such behavior in detail, as shown in Figs. $9 \mathrm{a}$ and $9 \mathrm{~b}$, indicates that the DSMC-PIC simulation offers a good foundation for including these effects.

Finally, in Figs. 10a and 10b, the localized ion currents collected on the horizontal barrels and on the vertical surfaces downstream (right hand side, RHS) and upstream (left hand side, LHS) of the accel and decel grids are shown at beam currents of 399 and $218 \mathrm{~mA}$, respectively. The abscissa is the computational cell number of each surface in either the horizontal (barrel) or vertical (RHS,LHS) directions. The accel grid data indicate that most of the ion current is collected on the horizontal barrel with a peak close to the upstream edge. For the decel grid, most of the ion current is collected on the downstream surface with a slight peak at the maximum radial coordinate where the pits and grooves are generated. These data support all of the conclusions discussed above drawn from the ion impact energy and angle distributions.

Another sensitive test of the accuracy of the model is the effect of increases in facility back pressure on the grid currents. These effects were measured experimentally at the Aerospace Corporation ${ }^{22}$ for thruster operating conditions of $\eta=0.80, \mathrm{~V}_{a c c}=-250 \mathrm{~V}$ and $-350 \mathrm{~V}$, and $\mathrm{V}_{d e c}=0 \mathrm{~V}$. The facility back pressure was increased by bleeding small amounts of xenon into the tank. Increases in back pressure above a level of about $5 \times 10^{-6}$ Torr produced discernible increases in accel grid current as shown in Fig. 11. Also included in Fig. 11 are data from the study of Bond and Latham ${ }^{13}$ for operating conditions of $\eta=0.80, V_{a c c}=-350 \mathrm{~V}$, $\mathrm{V}_{d e c}=0 \mathrm{~V}$. Finally, results from the present model are shown for conditions of $\mathrm{V}_{a c c}=-225 \mathrm{~V}$ and $\mathrm{V}_{d e c}=-50 \mathrm{~V}$. The small differences in grid voltages are not expected to be significant. Comparison of the data sets shows that excellent agreement between the present model and the experimental data is obtained. The results from Ref. 13 are also in good agreement with the measured data.

To obtain predictions of the total mass of grid eroded, the sputter yield for impact of xenon ions on molybdenum is integrated over the ion energy distributions such as those shown in Figs. 8a and 8b using the model described in Ref. 6. This model is based on the normal impact sputter yield of xenon ions on molybdenum measured by Weijsenfeld et al. ${ }^{23}$, together with a model for the angular dependence proposed by Yamamura et al.. ${ }^{24}$ The predictions of total grid mass eroded over 10,000 hours of operation for both the accel and decel grids are shown in Fig. 12. Comparison of these data with the accel and decel grid currents illustrates the importance of the energy and angle dependencies of the molybdenum sputter yield. Namely, while the ratio of accel to decel grid currents is generally about two, the ratio of accel to decel total mass erosion is as high as four. Thus, as would be expected from the results presented above, the higher average energy of ion impact on the accel grid leads to an increased rate of erosion in comparison to that on the decel grid above that due simply to the greater total ion current collected.

\section{Concluding Remarks}

Detailed computational analysis was performed of a single, representative aperture of the triple-grid T5 ion thruster. Overall effects for the 1,551 apertures of the $\mathbf{T} 5$ thruster were obtained by scaling up the results 
for the single aperture simulation. Comparisons of the computed ion currents collected on both the accel and decel grids revealed very good agreement over a range of thruster operating conditions. Distribution functions computed for the energy and incident angle of the ions impacting on the grids were used to understand in detail the nature of the impingement phenomena. These studies revealed that the accel grid ion current is mainly comprised of charge exchange ions created in the intergrid region that are pulled onto the accel grid because of its relatively low potential energy. There is also a significant fraction of ions that flow backwards from the plasma downstream of the decel grid onto the accel grid. For the decel grid ion current, almost all of this involves back-flowing ions. In comparison to the data for the decel grid, the greater average ion impact energy on the accel grid leads to an increase in grid mass erosion above that due only to the increased grid current.

\section{Acknowledgments}

The preparation of this manuscript was supported in part by The Aerospace Corporation through the Aerospace Independent Research and Development Program.

\section{References}

${ }^{1}$ Brophy, J.R., Polk, J.E., and Rawlin, V.K., "Ion Engine Service Life Validation by Analysis and Testing," AIAA Paper 96-2715, July 1996, and references therein.

${ }^{2}$ Crofton, M.W., "Evaluation of the United Kingdom Ion Thruster," Journal of Spacecraft and Rockets, Vol. 33, No. 5, 1996, pp. 739-747, and references therein.

${ }^{3}$ Brophy, J. R., et al., "Ion Propulsion System (NSTAR) DS1 Technology Validation Report," NASA JPL, Sept. 2000, (see http://nmp-techval-reports.jpl.nasa.gov/DS1/IPS_Integrated_Report.pdf).

${ }^{4}$ Kitamura, S., Hayakawa, Y., Kasai, Y., and Osaki, T., "Fabrication of Carbon-Carbon Composite Ion Thruster Grids-Improvement of Structural Strength," IEPC Paper 97-093, August 1997.

5 Soulas, G. C., Haag, T. W., Patterson, M. J., and Rawlin, V. K., "Titanium Optics for Ion Thrusters," IEPC Paper 99-149, October 1999.

${ }^{6}$ Boyd, I. D. and Crofton, M. W., "A Computational Study of Grid Erosion Through Ion Impact," AIAA Paper 2000-3664, July 2000.

${ }^{7}$ Birdsall, C. K. and Langdon, A. B., Plasma Physics Via Computer Simulation, Adam Hilger Press, 1991.

${ }^{8}$ Bird, G. A., Molecular Gas Dynamics and the Direct Simulation of Gas Flows, Oxford University Press, 1994.

${ }^{9}$ Crofton, M.W., and Boyd, I.D., "Origins of Accelerator Grid Current: Analysis of T5-Grid Test Results," Journal of Propulsion and Power, Vol. 17, 2001, pp. 203-211.

${ }^{10}$ Pollard, J.E., "Plume Angular, Energy, and Mass Spectral Measurements with the T5 Ion Engine," AIAA Paper 95-2920, July 1995.

11 Arakawa, Y. and Ishihara, K., "A Numerical Code for Cusped Ion Thrusters," IEPC Paper 91-118, October 1991. 
12 Peng, X., Keefer, D., and Ruyten, W., "Plasma Particle Simulation of Electro-static Ion Thrusters," Journal of Propulsion and Power, Vol. 8, No. 2, 1992, pp. 361-366.

13 Bond, R.A. and Latham, P.M., "Ion Thruster Extraction Grid Design and Erosion Modeling Using Computer Simulation," AIAA Paper 95-2923, July 1995.

14 Nakano, M. and Arakawa, Y., "Ion Thruster Lifetime Estimation and Modeling Using Computer Simulation," IEPC Paper 99-145, October 1999.

${ }^{15}$ De Boer, P.C.T., "Electric Probe Measurements in the Plume of an Ion Thruster," Journal of Propulsion and Power, Vol. 12, 1996, pp. 95-104.

16 Samanta Roy, R. I., Hastings, D. E., and Gatsonis, N. A., "Ion-Thruster Plume Modeling for Backflow Contamination," Journal of Spacecraft and Rockets, Vol. 33, No. 4, 1996, pp. 525-534.

17 Pullins, S., Chiu, Y., Levandier, D, and Dressler, R., "Ion Dynamics in Hall Effect and Ion Thrusters: $\mathrm{Xe}^{+}+\mathrm{Xe}$ Symmetric Charge Transfer," AIAA Paper 00-0603, January 2000.

${ }^{18}$ Dalgarno, A., McDowell, M. R. C., and Williams, A., "The Mobilities of Ions in Unlike Gases," Proceedings of the Royal Society, Vol. 250, 1958, pp. 411-425.

19 Nanbu, K., "Theory of Cumulative Small-Angle Collisions in Plasmas," Physical Review E, Vol. 55, No. 4, 1997, pp. 4642-4652.

20 Sadeghi, N., Nakano, T., Trevor, D. J., and Gottscho, R. A., "Ion Transport in an Electron Cyclotron Resonance Plasma," Journal of Applied Physics, Vol. 70, 1991, pp. 2552-2569.

21 Boyd, I. D. and Falk, M. J., "A Review of Spacecraft Material Sputtering by Hall Thruster Plumes," AIAA Paper 2001-3353, July 2001.

${ }^{22}$ Crofton, M. W. and Fearn, D. G., previously unpublished data, September 1994.

23 Weijsenfeld, C. H., Hoogendoorn, A., and Koedam, M., "Sputtering of Polycrystalline Metals by Inert Gas Ions of Low Energy (100-1000eV)," Physica, Vol. 27, 1961, p. 763.

24 Yamamura, Y., Itikawa, Y. and Itoh, N., "Angular Dependence of Sputtering Yields of Monatomic Solids," Report IPPJ-AM-26, Institute of Plasma Physics, Nagoya University, Japan, 1983.

Table 1. Operating data for grid set GS003.

\begin{tabular}{|c|c|c|c|c|c|c|c|c|c|}
\hline $\begin{array}{l}\mathrm{I}_{\text {beam }} \\
(\mathrm{mA})\end{array}$ & $\begin{array}{c}\dot{m} \\
(\mathrm{mg} / \mathrm{s})\end{array}$ & $\begin{array}{c}\eta \\
(-)\end{array}$ & $\begin{array}{c}\zeta \\
(-)\end{array}$ & $\begin{array}{c}\mathrm{V}_{\text {beam }} \\
(\mathrm{V})\end{array}$ & $\begin{array}{c}\mathrm{V}_{a c c} \\
(\mathrm{~V})\end{array}$ & $\begin{array}{c}\mathrm{V}_{d e c} \\
(\mathrm{~V})\end{array}$ & $\begin{array}{c}\mathrm{I}_{a c c} \\
(\mathrm{~mA})\end{array}$ & $\begin{array}{c}\mathrm{I}_{d e c} \\
(\mathrm{~mA})\end{array}$ & $\begin{array}{c}\mathbf{F} \\
(\mathrm{mN})\end{array}$ \\
\hline 218 & 0.359 & 0.754 & 0.170 & 1100 & -225 & -50 & 0.53 & 0.25 & 11.9 \\
\hline 270 & 0.482 & 0.720 & 0.105 & 1100 & -225 & -50 & 0.90 & 0.44 & 14.8 \\
\hline 327 & 0.626 & 0.685 & 0.070 & 1100 & -225 & -50 & 1.43 & 0.69 & 17.9 \\
\hline 328 & 0.551 & 0.759 & 0.126 & 1100 & -225 & -50 & 1.10 & 0.46 & 17.9 \\
\hline 348 & 0.551 & 0.790 & 0.161 & 1100 & -225 & -50 & 1.05 & 0.38 & 19.0 \\
\hline 363 & 0.626 & 0.751 & 0.094 & 1100 & -225 & -50 & 1.37 & 0.55 & 19.9 \\
\hline 399 & 0.626 & 0.798 & 0.159 & 1100 & -225 & -50 & 1.25 & 0.40 & 21.8 \\
\hline
\end{tabular}




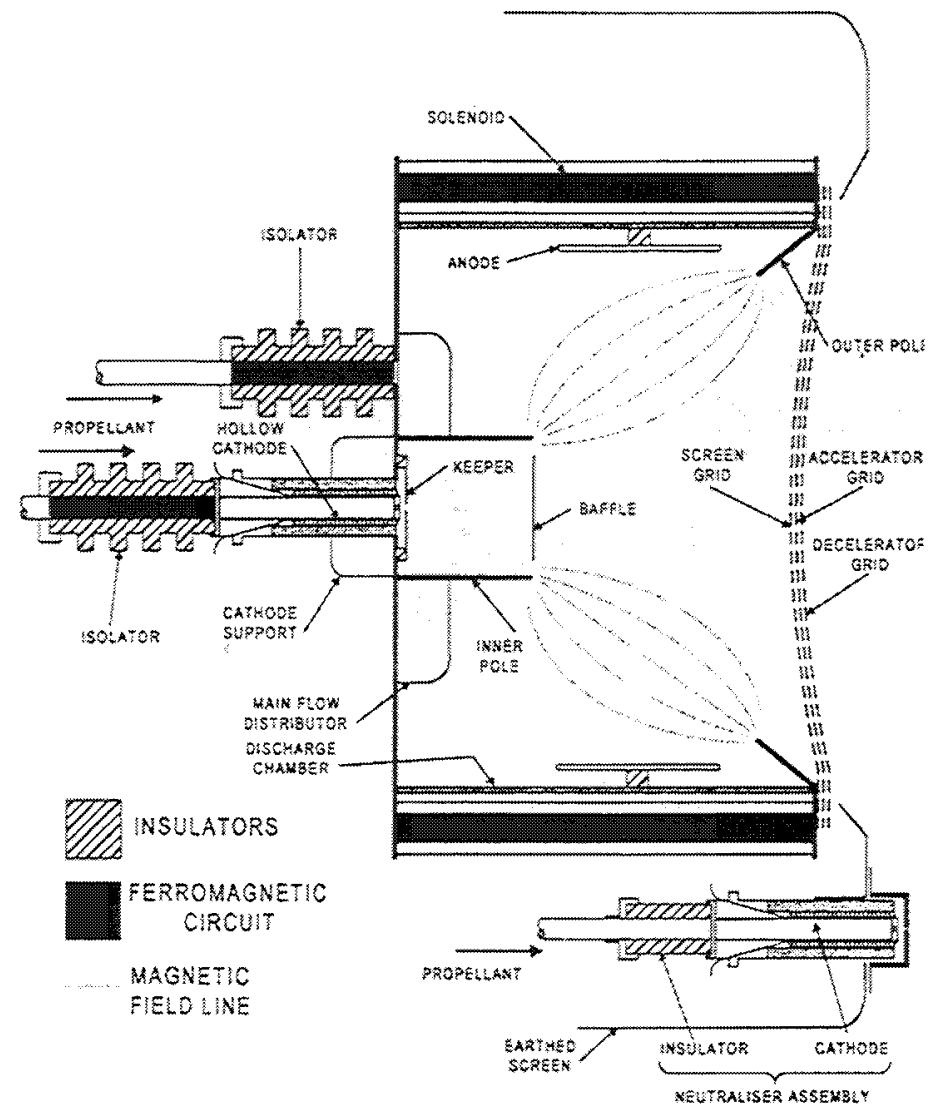

Fig. 1. Schematic diagram of the UK-T5 thruster.

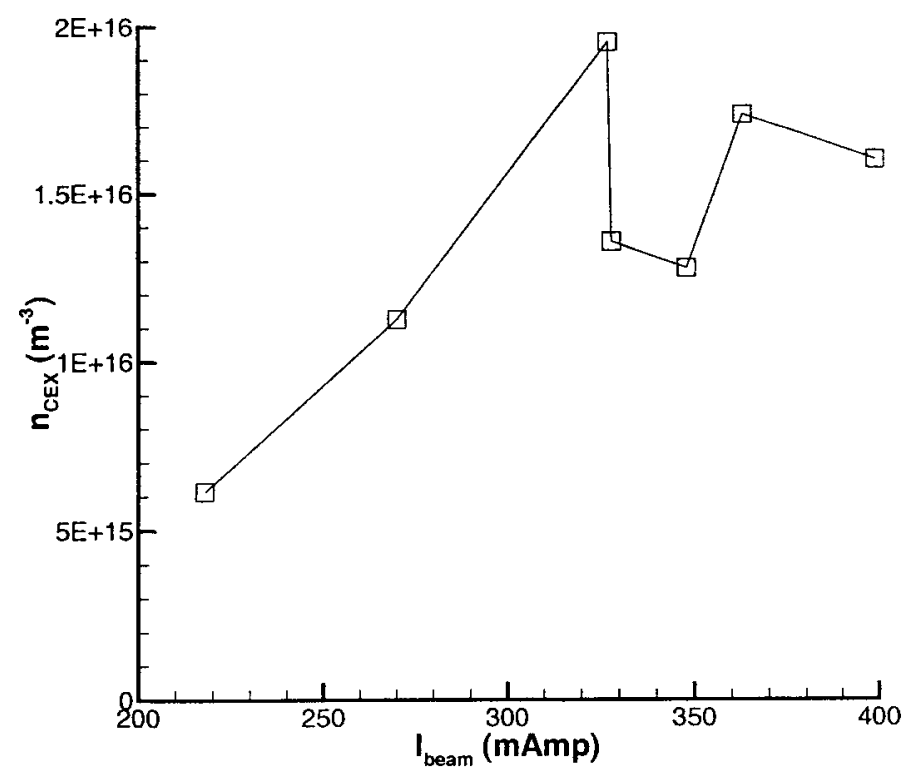

Fig. 2. Reference electron densities as a function of beam current. 


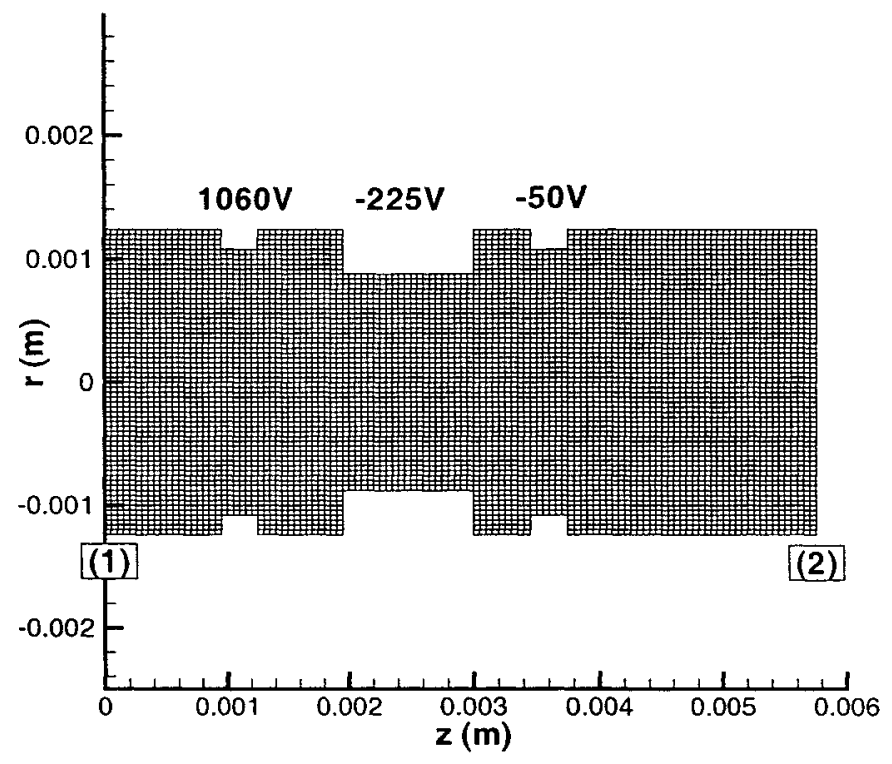

Fig. 3. Computational grid.

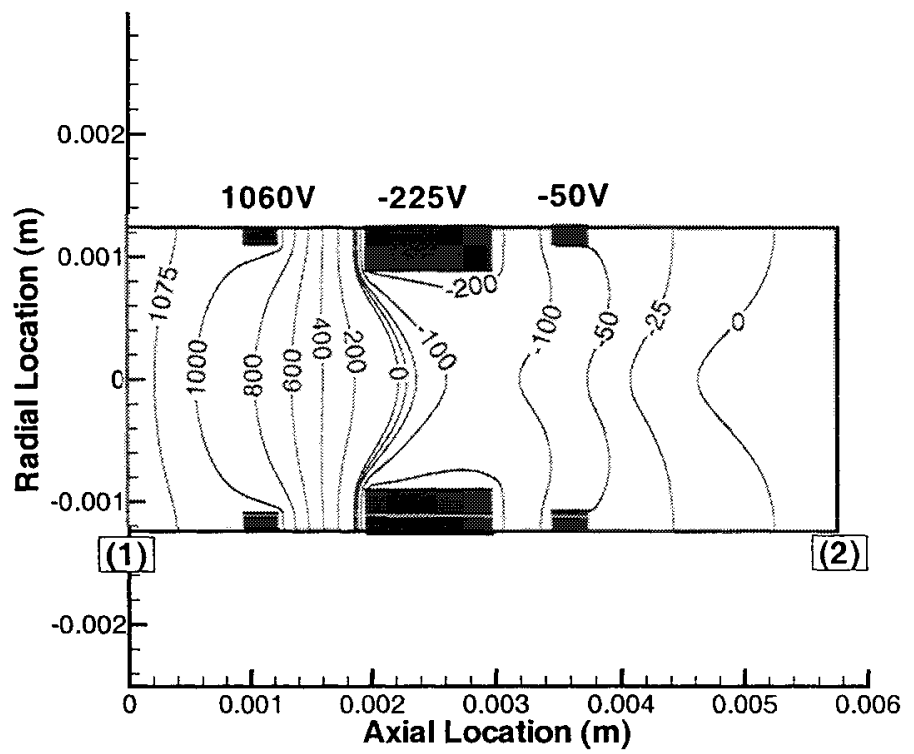

Fig. 4. Contours of plasma potential. 


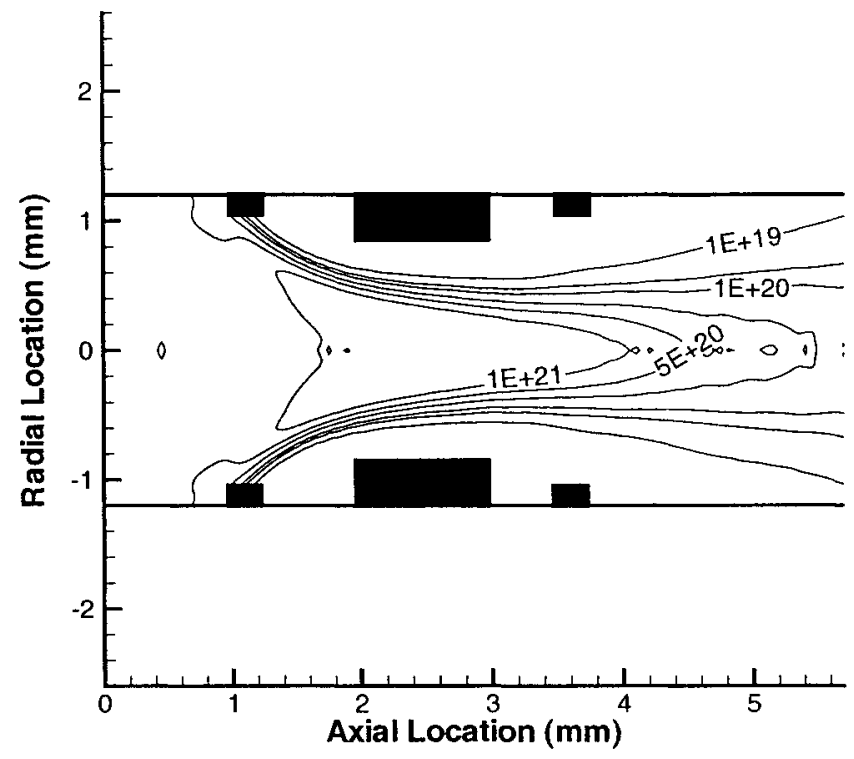

Fig. 5. Contours of volumetric production rate of charge exchange ions $\left(\mathrm{m}^{-3} \mathrm{~s}^{-1}\right)$.

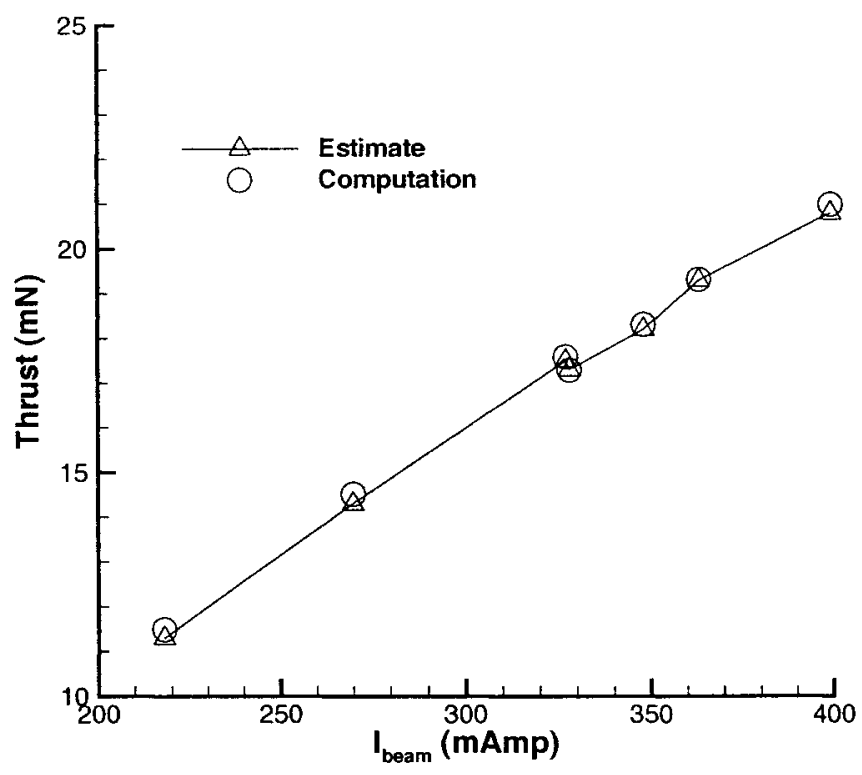

Fig. 6. Thrust as a function of beam current. 


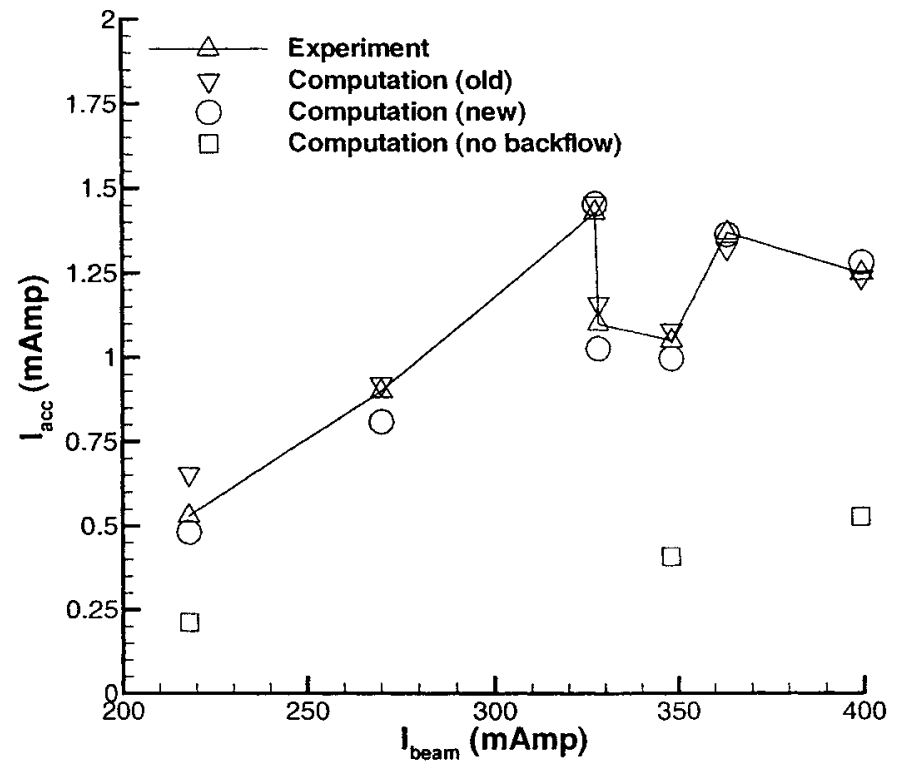

Fig. 7a. Current collected on the accel grid as a function of beam current.

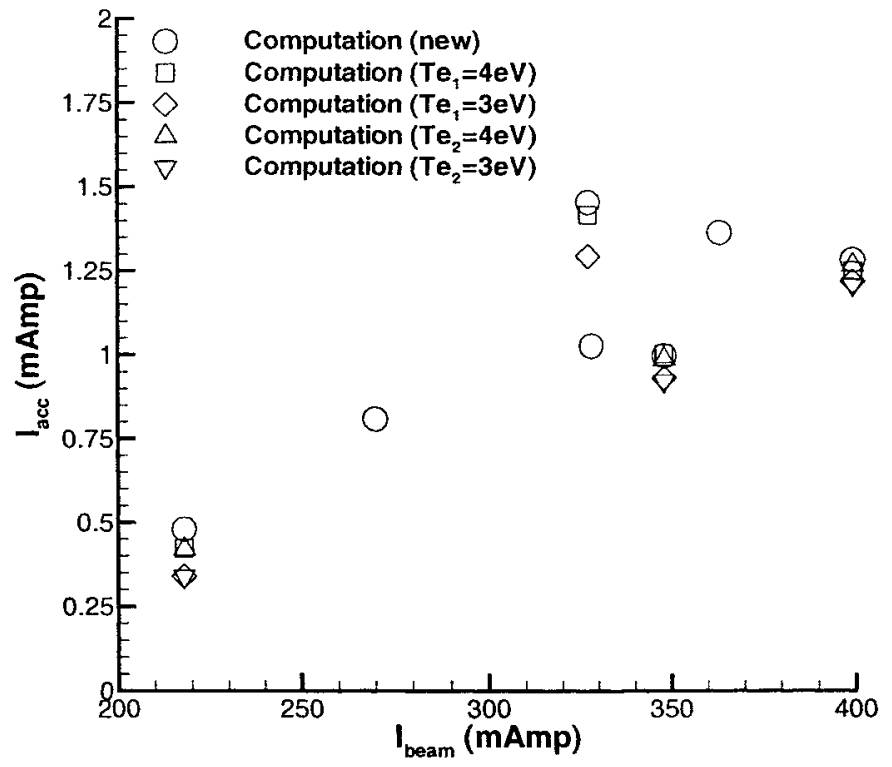

Fig. 7b. Current collected on the accel grid: sensitivity to assumed plasma properties. 


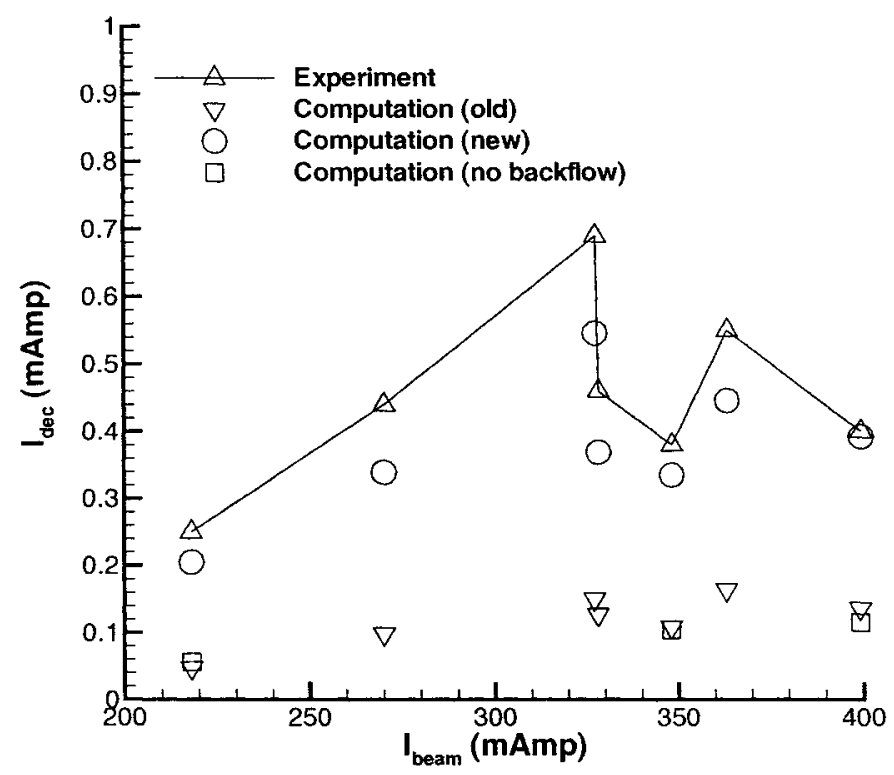

Fig. 7c. Current collected on the decel grid as a function of beam current.

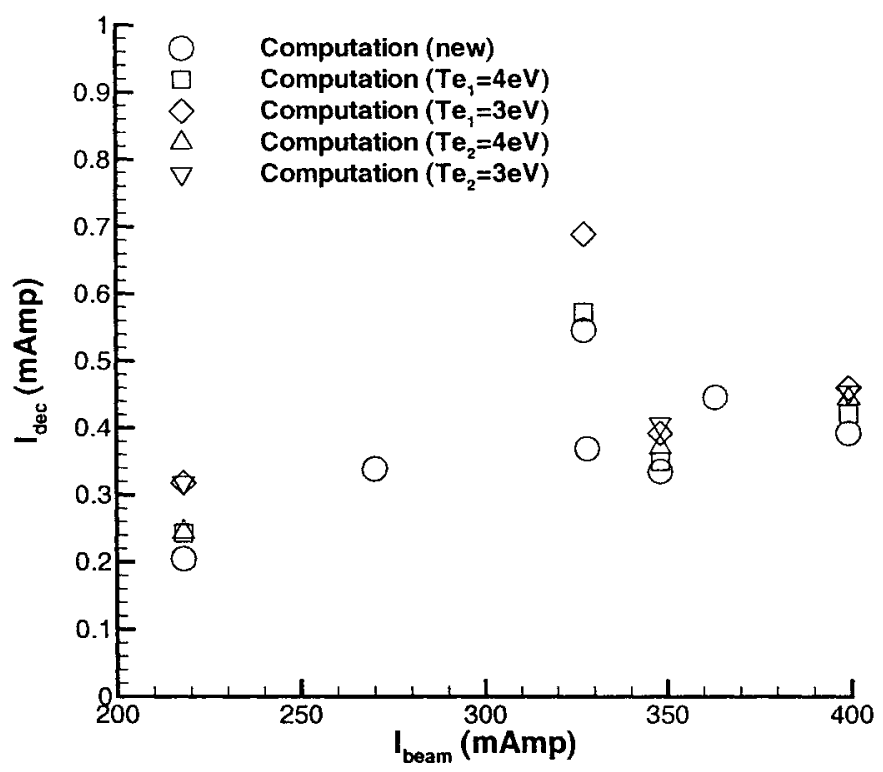

Fig. 7 d. Current collected on the decel grid: sensitivity to assumed plasma properties. 


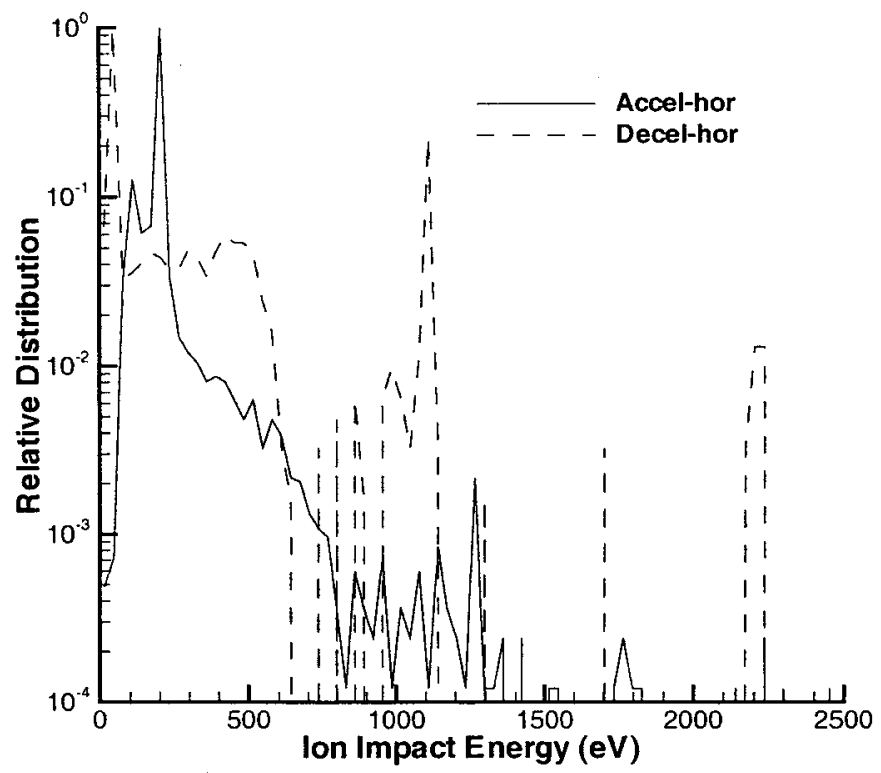

Fig. 8a. Ion energy distributions on the barrel of the accel and decel grids at $399 \mathrm{~mA}$ beam current.

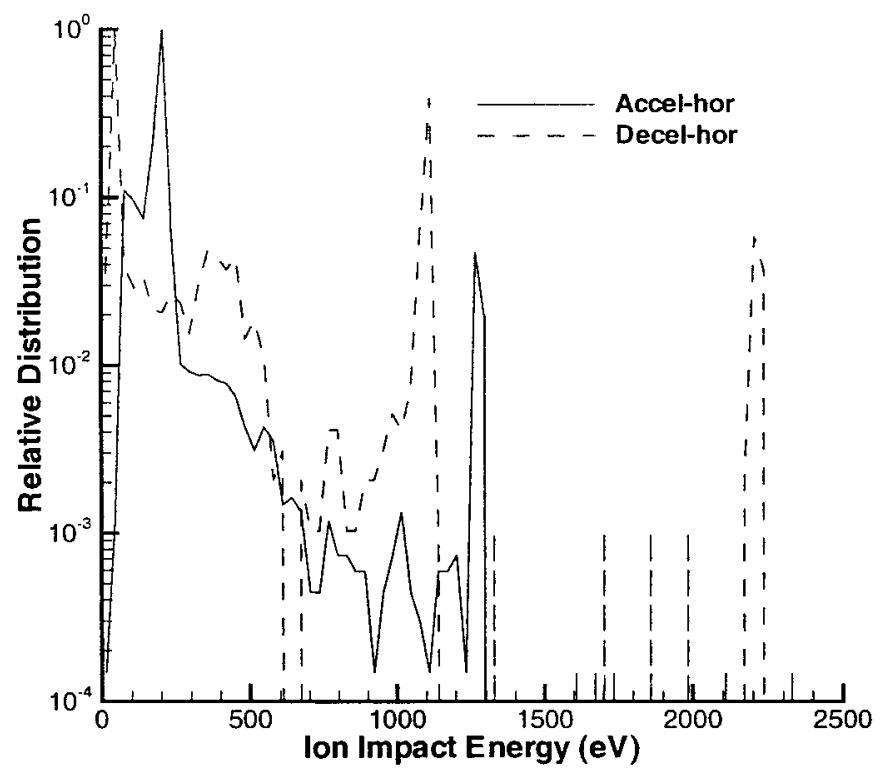

Fig. 8b. Ion energy distributions on the barrel of the accel and decel grids at $218 \mathrm{~mA}$ beam current. 


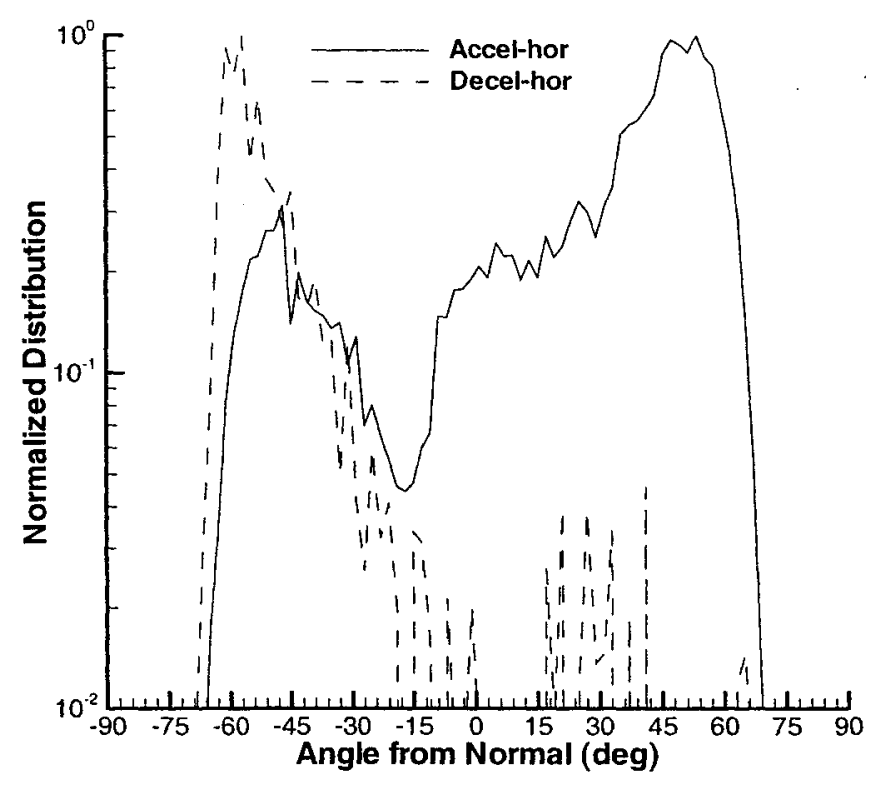

Fig. 9a. Ion impact angle distributions on the barrel of the accel and decel grids at $399 \mathrm{~mA}$ beam current.

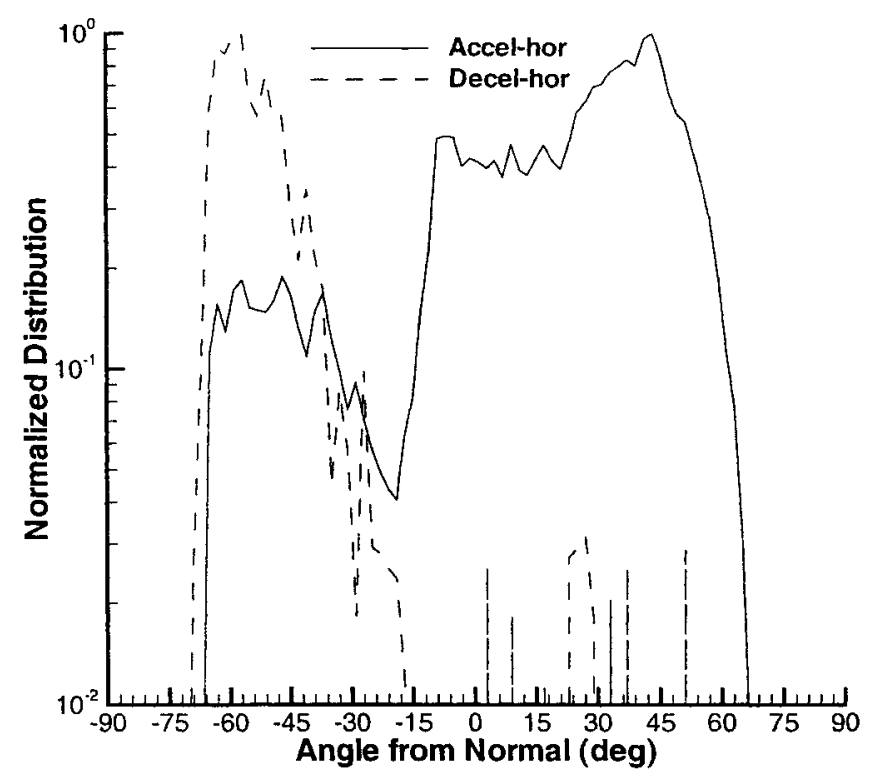

Fig. 9b. Ion impact angle distributions on the barrel of the accel and decel grids at $218 \mathrm{~mA}$ beam current. 


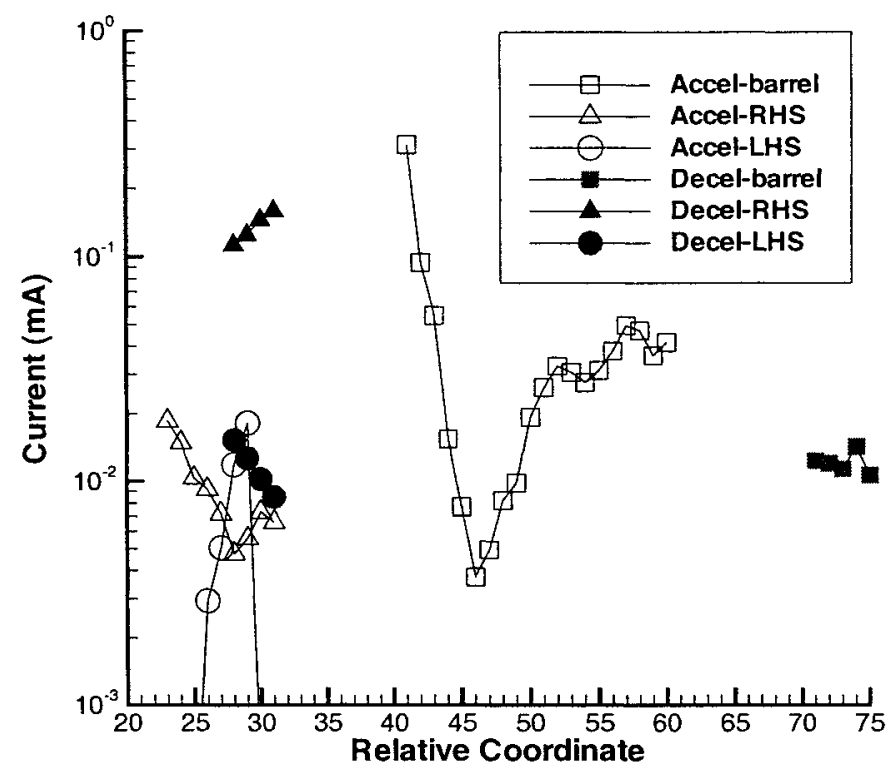

Fig. 10a. Ion current on surfaces of the accel and decel grids at $399 \mathrm{~mA}$ beam current.

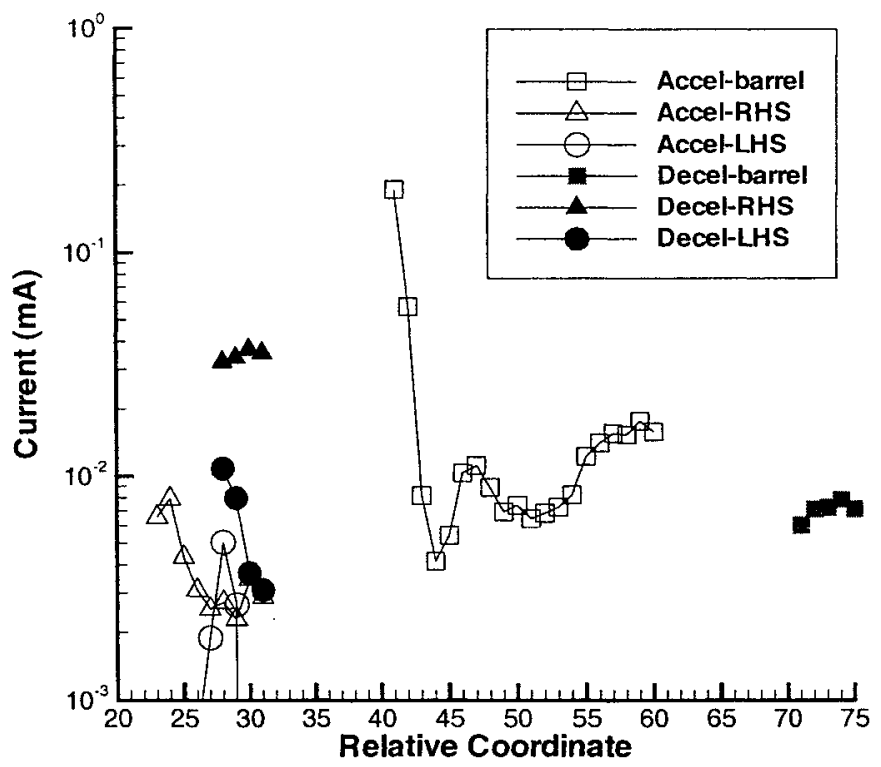

Fig. 10b. Ion current on surfaces of the accel and decel grids at $218 \mathrm{~mA}$ beam current. 


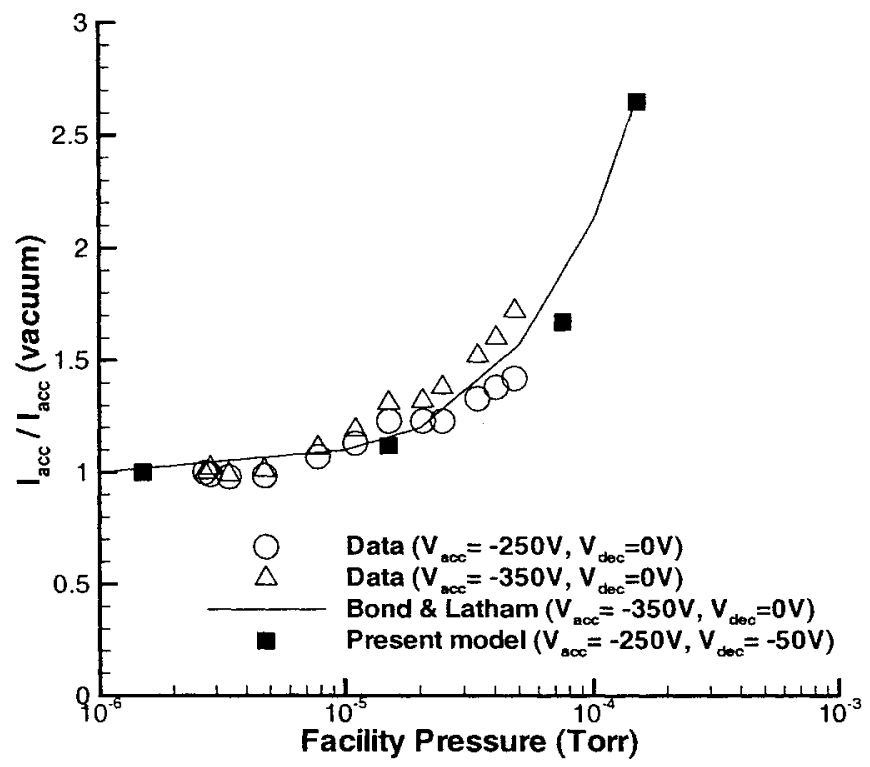

Fig. 11. Effect of facility back pressure on the accel grid current for the UK-T5 thruster.

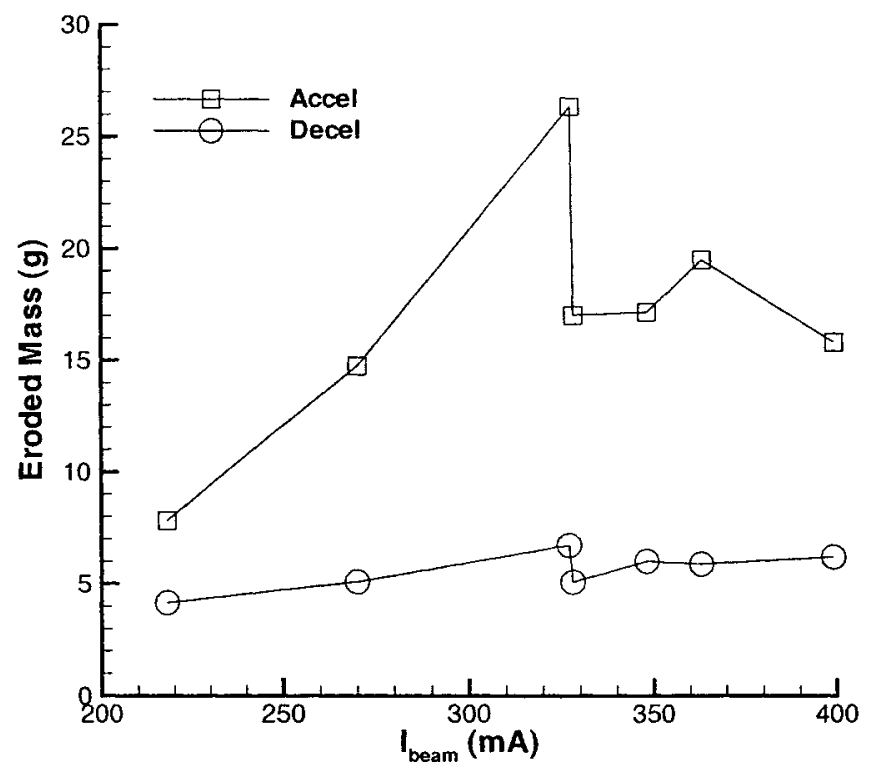

Fig. 12. Predictions for total mass erosion of the accel and decel grids for 10,000 hours of operation. 